\section{Abnormal movement of the arytenoid region as a cause of upper airway obstruction}

Atsushi Nagai, Toshinori Kanemura, Kimio Konno

\begin{abstract}
A 75 year old woman presented with a three week history of severe dyspnoea and cough. Auscultation and spirometry suggested extrathoracic inspiratory airway obstruction, and bronchoscopy showed abnormal motion of the arytenoid region (supraglottic area), causing upper airway obstruction only during forced inspiratory efforts. Sedatives improved the symptoms within a week. It is suggested that reversible malfunction of the arytenoid region can be responsible for upper airway obstruction.
\end{abstract}

(Thorax 1992;47:840-841)

Cases of reversible (functional) upper airway obstruction are increasingly being reported, most of which are due to abnormal motion of the vocal cords..$^{1-6} \mathrm{We}$ have previously reported a case of functional pharyngeal constriction as a cause of inspiratory stridor. ${ }^{7}$ The present patient had inspiratory airflow obstruction due to abnormal motion within the arytenoid region, which has not been previously described.

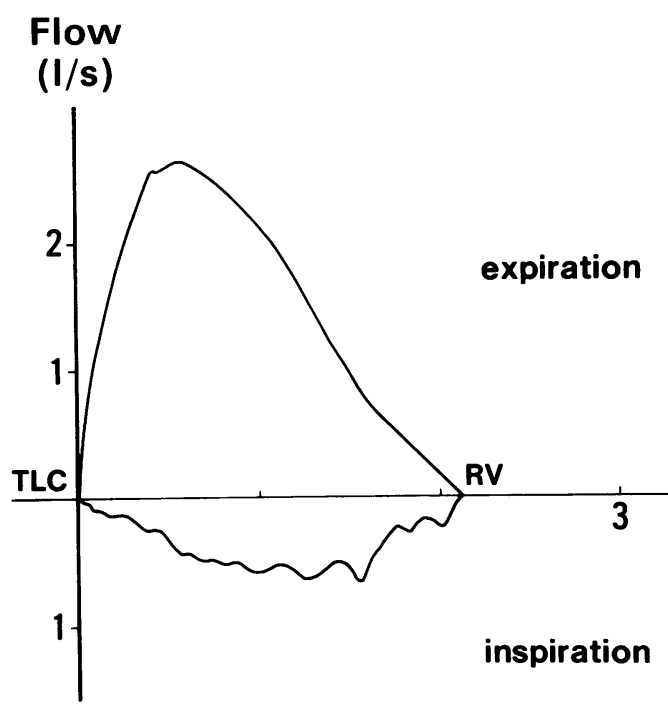

Figure 1 Flow-volume loop, showing near normal expiratory flow and a decrease in inspiratory flow at any forced inspiratory volume. TLC-total lung capacity; forced inspiratory volume
$R V-$ residual volume.

\section{Case report}

A 75 year old woman presented with a three week history of severe dyspnoea after coughing. She did not have a history of bronchial asthma, respiratory infections, or allergies. There was domestic discord in the family and she had suffered from insomnia for several months. Physical examination showed prolonged inspiration with severe stridor during forced inspiratory effort. Spirometry showed a substantial decrease in forced inspiratory flow with a plateau configuration but a more normal expiratory flow pattern, suggesting extrathoracic airway obstruction (fig 1). Immediately after spirometry fibreoptic bronchoscopy examination was performed on the patient without local anaesthesia or sedation, and forced inspiratory efforts made the arytenoid region move to obstruct the laryngeal orifice (fig 2 ). This abnormal motion of the arytenoid region did not occur during quiet respiration. The pharynx, glottis, vocal cords, and trachea were normal. Chest radiography, electrocardiography, and routine laboratory tests (including determination of $\mathrm{IgE}$ concentration) all gave normal results. Neurological examin-

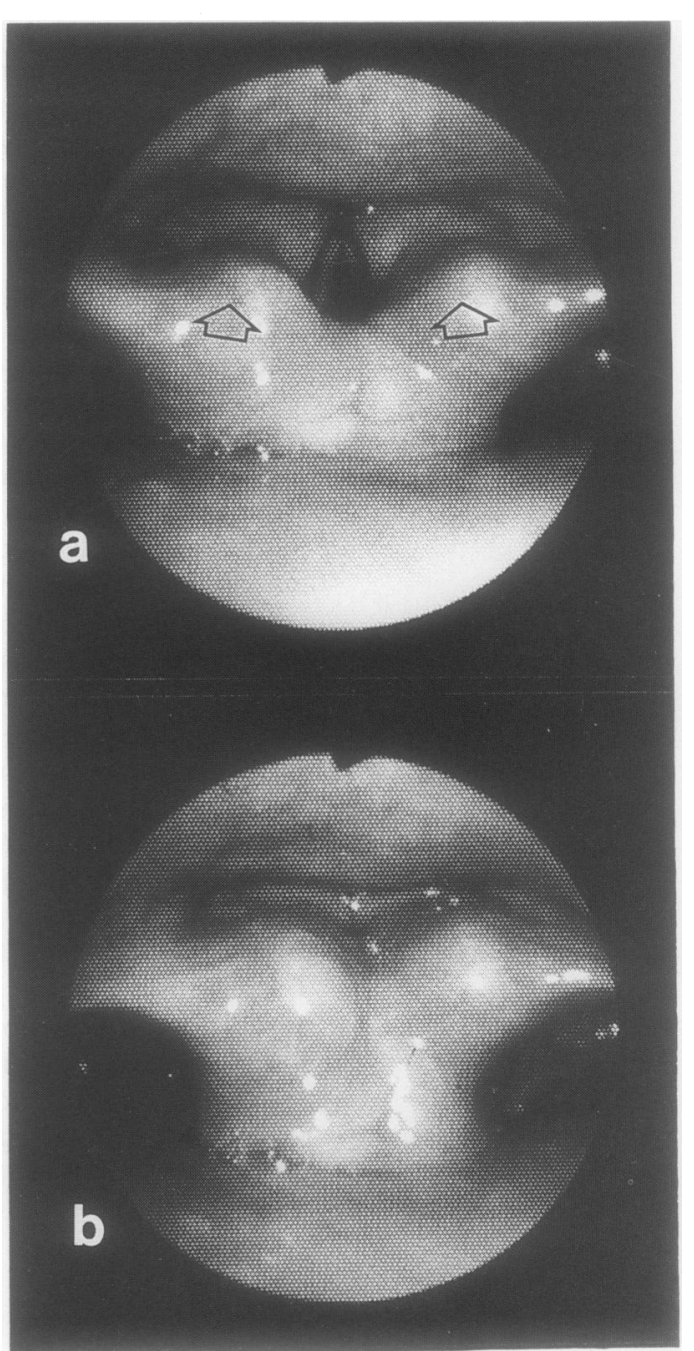

Figure 2 Bronchoscopic findings during forced inspiratory effort showing narrowing in the laryngeal orifice due to abnormal movement of the arytenoid region (arrows). The orifice is narrowing ( $a$ ) at the beginning of the inspiratory phase and then is completely closed (b). 
ation and electroencephalography showed no abnormalities. A psychiatric evaluation suggested emotional stress. She was treated with $6 \mathrm{mg}$ diazepam/day. After one week her complaints disappeared and there was no inspiratory stridor even when she inspired as strongly as possible. At that time repeat bronchoscopy was performed and the abnormal motion of the arytenoid region was not observed. Since then she has received sedative treatments infrequently and has had no further complaints in four months of follow up.

\section{Discussion}

We present a case with inspiratory dyspnoea induced by abnormal motion of the arytenoid region. Reversible upper airway obstruction is rare in adolescents. In 1974 upper airway obstruction without organic abnormalities was described by Patterson et al, ${ }^{8}$ and since then several other cases have been reported. Abnormal (paradoxical) movement of the vocal cords is the most frequent cause of upper airway obstruction. This patient showed abnormal motion of the arytenoid region during forced inspiratory efforts but no abnormality in the pharynx, vocal cords, or trachea and had no laryngeal spasm.

The association of coughing and abnormal motion of the arytenoid region is hard to explain. The patient had no history of asthma or other allergic conditions. In view of her sudden onset of symptoms without any organic disease and of her emotional stress she was given sedatives, and one week later the abnormal motion as well as the coughing attacks had disappeared. Thus these symptoms may have been due to a psychogenic or undetectable neurogenic problem. Finally, we suggest that abnormal motion of the arytenoid region may be an additional cause of upper airway obstruction, and that direct fibrescopic observations and forced expiratory and inspiratory flowvolume loops effectively reveal such a cause of upper airway obstruction.

1 Rogers JH, Stell PM. Paradoxical movement of the vocal cords as a cause of stridor. J Laryngol Otol 1978;92:157-8.

2 Kellmann RM, Leopold DA. Paradoxical cord motion: an important cause of stridor. Laryngoscope 1982;92:58-60.

3 Appelblatt NH, Baker SR. Functional upper airway obstruction: a new syndrome. Arch Otolaryngol 1981;107: 305-6.

4 Ophir D, Katz Y, Tavori I, Aladjen M. Functional upper airway obstruction in adolescents. Arch Otolaryngol Head Neck Surg 1990;116:1208-9.

5 Kattan M, Ben-Zvi Z. Stridor caused by vocal cord malfunction associated with emotional factors. Clin Pediatr (Philadelphia) 1985;24:158-60.

6 Christopher KL, Wood RP, Eckert C, Blager FB, Raney RA, Souhrada JF. Vocal cord dysfunction presenting as asthma. N Engl J Med 1983;308:1566-70.

7 Nagai A, Sakamoto K, Yamaguchi E, Takahashi E. Functional upper airway obstruction: psychogenic pharyngeal constriction. Chest 1992;101:1460-1.

8 Patterson R, Schatz M, Horton M. Munchausen's stridor: non-organic laryngeal obstruction. Clin Allergy 1974; 4:307-10. 